\title{
ON THE OPTIMAL COMBINATION OF LEVELING NETWORKS
}

\author{
Dimitrios AMPATZIDIS ${ }^{1)} *$, Rolf KOENIG ${ }^{1)}$ and Nikolaos DEMIRTZOGLOU ${ }^{2)}$ \\ ${ }^{1)}$ GFZ German Centre for Geosciences, c/o DLR Oberpfaffenhofen, 82234 Wessling, Germany, \\ ${ }^{2)}$ Freelance Surveyor Engineer, Areos Street 22, Drama, Greece, 66100 \\ *Corresponding author's e-mail: ampatzi@gfz-potsdam.de
}

\begin{tabular}{l} 
ARTICLE INFO \\
\hline Article history: \\
Received 8 January 2015 \\
Accepted 9 July 2015 \\
Available online 31 July 2015 \\
\hline
\end{tabular}

\section{Keywords:}

Datum

Leveling network

Optimal combination

\begin{abstract}
Accurate heights are needed in engineering and geodynamics. The classical technique of height determination is spirit leveling. In recent years GNSS-leveling offers an efficient alternative. The paper deals with the combination of existing leveling networks. In many cases, in a specified area more than one leveling network exist, realized at different epochs and based on different techniques and instrumentation. Hence, the surveyor should have the capability to exploit all the existing height information in order to establish an optimal network, i.e. the most accurate one. We describe a methodology for the optimal combination of vertical networks when these share some common benchmarks. Its advantage is the fact that all the existing height information can eventually be transferred to a new vertical network that is more accurate than the individual networks. The approach is tested on three co-located leveling networks (one trigonometric network, two spirit leveling ones) in a regional area located in Greece. The results reveal a significant improvement of $5 \mathrm{~mm}$ of the standard deviations of the height estimates compared to the prior situation.
\end{abstract}

\section{INTRODUCTION}

Spirit leveling can reach sub-mm accuracy depending on the length of the leveling line. Over long distances GNSS-leveling becomes superior to spirit leveling. This is the reason why e.g. the distortions of the North American height system could be detected (e.g., Vanicek and Krakiwsky, 1982; Torge, 2001). During the last decades, the availability of GNSS techniques changed drastically the concept of geometric height determination. The cumbersome spirit leveling measurements are gradually replaced by the GNSS leveling (e.g., Hofmann-Wellenhof and Moritz, 2005). The usefulness of GNSS-leveling for the determination of normal, dynamic or orthometric heights is strongly dependent on the quality of the available gravimetric geoid. If one seeks for geometric vertical positioning, GNSS leveling would be the method of choice without having to perform cumbersome spirit leveling together with gravity measurements; see Wang et al. (2012).

Numerous studies and models are implemented in the direction of GNSS leveling theoretical background and practical realization (e.g., Kotsakis and Sideris, 1999; Fotopoulos, 2003; Hirt et al., 2011). The main concept of the GNSS leveling is based on the simultaneous use of different kinds of heights: ellipsoidal heights from GNSS and geoidal/quasi geoidal heights from gravity measurements and global geopotential models to derive orthometric or normal heights as they result from spirit leveling. A crucial problem of orthometric/normal height systems is that their datums differ significantly from country to country, or even from region to region within each country. E.g the Greek vertical networks present great inconsistencies and systematic effects. The main reason of this problem is, that the height reference system has not been unified; see e.g, (Kotsakis et al., 2012). In addition, the height information of the benchmarks of the triangulation network of the state is not consistent to that of the stations of the official vertical network. Till now there is no official study on the quantification of the vertical datum discrepancies.

When a classical leveling campaign is organized and implemented in the field, it is possible to find height benchmarks from previous realizations of vertical networks. These benchmarks are normally endowed with the height value itself (referring to some datum) and its associated accuracy. A straightforward procedure for the surveyor is to remeasure the existing benchmarks and to quantify and qualify the vertical offsets between the new and the old campaigns respectively. Significant offsets can be attributed to different datum choices, to instrumental systematic biases, or even to gross errors in the observations or computations. In the majority of all cases we do not have the observation material of the older campaigns, and the information about the choices of the datum and about other network constraints is missing. Hence, there is a need to introduce a proper mathematical tool in order to exploit and assimilate all existing height information, old and new, in an optimal way. By optimal we mean that the combination leads to the most accurate 
heights that can be determined based on the available information.

The present paper deals with a new methodology for the optimal combination of vertical networks. We formulate all the necessary mathematical background for this purpose and the new technique is implemented and tested based on real data in an area located in the Northern part of Greece.

\section{THE MATHEMATICAL MODEL}

We may distinguish between three situations that arise in practice in relation to the records of past leveling network solutions:

(a) The complete solution is available, that is the height estimates and their complete covariance matrix.

(b) The height estimates are available together with their variances, i.e. the diagonal part of the covariance matrix only is known.

(c) The height estimates only are available.

Solely in case (a) we may obtain a statistically optimal solution for the combination of the leveling networks. The algorithm presented here applies to the sub-optimal solution of case (b) where only the variances of the estimated heights are known, without any covariance information. For the case (c) all one can do is to assume that all heights within each network have the same variance and no correlations.

Suppose that we have $r$ different vertical networks and their associated formal errors of the estimated height parameters. In addition, let us consider that in those networks there is a number of common points. Our main concern is to define the vertical optimal network, implementing an alternative approach for their combination. The new strategy relies on the appropriate reconstruction of the normal equations of each network and their combination for the final solution. For each of the $k$ vertical networks , we can write the following mathematical expression:

$h_{1}^{k}=h_{k, 1}+t^{k}+e_{1}^{k}$

$h_{n_{k}}^{k}=h_{k, n_{k}}+t^{k}+e_{n_{k}}^{k}$

$k=1,2, \ldots, r$

or, in a more compact matrix form:

$\mathbf{h}^{k}=\mathbf{h}_{k}+\mathbf{1}_{n_{k}} t^{k}+\mathbf{e}_{k}$

where $n_{k}$ is the number of points in the $k$ network and $r$ the number of the networks to be combined. The vector $\mathbf{h}^{k}$ contains all the observed heights of the $k$ network. The vector $\mathbf{1}_{n_{k}}=\left[\begin{array}{lll}1 & \cdots & 1\end{array}\right]^{T}$ has length equal to $n_{k}$. The vector $\mathbf{h}_{k}$ contains the unknown heights of all points of the $k$ network and at least one of them are shared by another network and $t^{k}$ is the shift term connecting the heights of the $k^{\text {th }}$ network to the optimal one. The term $\mathbf{e}_{k}$ refers to the height errors of the $k$ network. In order to proceed to the final solution, we shall separate the algorithm into three sequential steps.

From the network design aspect, it will be hardly the case that all networks have a common overlapping part. The combined networks may overlap in pairs. A more common situation will be that of a new GNSS leveling network, which overlaps with any of the old traditional networks, while the latter are completely disjoint. A more extreme situation may arise if all points of an ensemble of old traditional networks that are completely disjoint are being re-observed by GNSS leveling. Our algorithm adjusts all heights of all networks in combination and not just the common parts (which may even be void as explained above) to account for the implicit correlations. We expand the algorithm into three steps:

\section{FIRST STEP: THE ESTIMATION OF THE INNER CONSTRAINTS SOLUTION}

The information that is available are the heights of each network (denoted as $\mathbf{h}_{k}$ ) and their associated standard deviations. The minimum constrained solution $\tilde{\mathbf{h}}_{k}$ differs from any other solution $\mathbf{h}_{k}$ by the shift term $t^{k}$, which can be expressed with the following formula:

$\tilde{\mathbf{h}}^{k}=\mathbf{h}^{k}+\mathbf{1}_{n_{k}} t^{k}$

The derivation of the so called inner constraints approach (see Blaha, 1971; Dermanis, 1987; Koch, 1999) is realized implementing the following optimal criterion:

$\phi=\left(\mathbf{h}_{E}^{k}\right)^{T} \mathbf{h}_{E}^{k}=\left(\mathbf{h}^{k}+\mathbf{1}_{n_{k}} t^{k}\right)^{T}\left(\mathbf{h}^{k}+\mathbf{1}_{n_{k}} t^{k}\right)=\min$

where $\mathbf{h}_{E}^{k}$ stands for the heights derived from the inner constraints solutions. Therefore $\phi$ means that the norm of the adjusted heights becomes minimum one. The solution of Eq. (4) is obtained as follows:

$$
\begin{gathered}
\frac{\partial \phi}{\partial t^{k}}=\frac{\partial\left(\mathbf{h}^{k}+\mathbf{1}_{n_{k}} t^{k}\right)^{T}\left(\mathbf{h}^{k}+\mathbf{1}_{n_{k}} t^{k}\right)}{\partial t^{k}}= \\
2\left(\mathbf{h}^{k}+\mathbf{1}_{n_{k}} t^{k}\right)^{T} \mathbf{1}_{n_{k}}=0 \Rightarrow \\
t^{k}=-\left(\mathbf{1}_{n_{k}}^{T} \mathbf{1}_{n_{k}}\right)^{-1} \mathbf{1}_{n_{k}}^{T} \mathbf{h}^{k}
\end{gathered}
$$

The new inner constrained solution reads:

$$
\begin{aligned}
\mathbf{h}_{E}^{k} & =\mathbf{h}^{k}+\mathbf{1}_{n_{k}} t^{k}=\mathbf{h}^{k}-\mathbf{1}_{n_{k}}\left(\mathbf{1}_{n_{k}}^{T} \mathbf{1}_{n_{k}}\right)^{-1} \mathbf{1}_{n_{k}}^{T} \mathbf{h}^{k}= \\
& =\left[\mathbf{I}_{n_{k}}-\frac{1}{n_{k}} \mathbf{1}_{n_{k}} \mathbf{1}_{n_{k}}^{T}\right] \mathbf{h}^{k}
\end{aligned}
$$


The full covariance matrix $\mathbf{C}_{k}^{E}$ of $\mathbf{h}_{E}^{k}$ can be easily derived, implementing the propagation law, as follows:

$$
\begin{aligned}
& \mathbf{C}_{k}^{E}=\left[\mathbf{I}_{n_{k}}-\frac{1}{n_{k}} \mathbf{1}_{n_{k}} \mathbf{1}_{n_{k}}^{T}\right] \mathbf{C}_{k}\left[\mathbf{I}_{n_{k}}-\frac{1}{n_{k}} \mathbf{1}_{n_{k}} \mathbf{1}_{n_{k}}^{T}\right]^{T}=\mathbf{C}_{k}-\frac{1}{n_{k}} \mathbf{C}_{k} \mathbf{1}_{n_{k}} \mathbf{1}_{n_{k}}^{T}-\frac{1}{n_{k}} \mathbf{1}_{n_{k}} \mathbf{1}_{n_{k}}^{T} \mathbf{C}_{k}+\frac{\mathbf{1}_{n_{k}}^{T} \mathbf{C}_{k} \mathbf{1}_{n_{k}}}{n_{k}^{2}} \mathbf{1}_{n_{k}} \mathbf{1}_{n_{k}}^{T} \Rightarrow \\
& \mathbf{C}_{k}^{E}=\mathbf{C}_{k}+\frac{1}{n_{k}^{2}}\left(\mathbf{1}_{n_{k}}^{T} \mathbf{C}_{k} \mathbf{1}_{n_{k}}-n_{k}\left(\mathbf{C}_{k} \mathbf{1}_{n_{k}} \mathbf{1}_{n_{k}}^{T}+\mathbf{1}_{n_{k}} \mathbf{1}_{n_{k}}^{T} \mathbf{C}_{k}\right)\right)
\end{aligned}
$$

where $\mathbf{C}_{k}$ is the (diagonal) error covariance matrix of the $k$ network heights.

One can immediately think to invert the covariance matrix $\mathbf{C}_{k}^{E}$ in order to derive the Normal Equation matrix $\mathbf{N}_{k}$. However, the matrix $\mathbf{C}_{k}^{E}$ is not invertible, since the associated covariance matrix of the inner constraints approach is a pseudoinverse matrix (thus singular). At this point, we shall recall the inner constraints covariance matrix formulation (e.g, Dermanis, 1987):

$$
\mathbf{C}_{k}^{E}=\left(\mathbf{N}_{k}+\mathbf{1}_{n_{k}} \mathbf{1}_{n_{k}}^{T}\right)^{-1}-\mathbf{1}_{n_{k}}\left(\mathbf{1}_{n_{k}}^{T} \mathbf{1}_{n_{k}}\right)^{-2} \mathbf{1}_{n_{k}}^{T}=\left(\mathbf{N}_{k}+\mathbf{1}_{n_{k}} \mathbf{1}_{n_{k}}^{T}\right)^{-1}-\frac{1}{n_{k}^{2}} \mathbf{1}_{n_{k}} \mathbf{1}_{n_{k}}^{T}
$$

Combining Eq. (7) and (8) we get the associated Normal Equation matrix of the $k^{\text {th }}$ network:

$$
\mathbf{N}_{k}=\left(\mathbf{C}_{k}^{E}+\frac{1}{n_{k}^{2}} \mathbf{1}_{n_{k}} \mathbf{1}_{n_{k}}^{T}\right)^{-1}-\mathbf{1}_{n_{k}} \mathbf{1}_{n_{k}}^{T}
$$

Then, the right hand term

$\mathbf{u}_{k}=\mathbf{N}_{k} \mathbf{h}_{k}$

is directly computed from the already known matrices $\mathbf{N}_{k}$ and $\mathbf{h}_{k}$.

\section{THIRD STEP: THE ADDITION OF NORMAL EQUATIONS FOR THE FINAL NETWORK SOLUTION}

In the final step, we compute the necessary matrices for the total adjustment. The final Normal Equation matrix will be:

$$
\mathbf{N}=\left[\begin{array}{cccccc}
\sum_{k=1}^{r} \dot{\mathbf{N}}_{k} & \tilde{\mathbf{N}}_{m 1} & \tilde{\mathbf{N}}_{m 2} & \cdots & \cdots & \tilde{\mathbf{N}}_{m N_{k}} \\
\tilde{\mathbf{N}}^{T} & \mathbf{N}_{1} & \mathbf{0} & \cdots & \cdots & \mathbf{0} \\
\tilde{\mathbf{N}}^{T}{ }^{T} & \mathbf{0} & \mathbf{N}_{2} & \mathbf{0} & \cdots & \mathbf{0} \\
\vdots & \vdots & \mathbf{0} & \ddots & \cdots & \vdots \\
\vdots & \vdots & \vdots & \vdots & \ddots & \vdots \\
\tilde{\mathbf{N}}^{T} & \mathbf{0} & \mathbf{0} & \mathbf{0} & \mathbf{0} & \mathbf{N}_{N_{k}}
\end{array}\right]
$$

where $\dot{\mathbf{N}}_{k}, k=1 \ldots r$ is the sub-matrix containing the information for the $m$ common points for each network $k$, $\mathbf{N}_{1}, \ldots, \mathbf{N}_{N_{k}}$ stands for the part consisting of the elements regarding the non-common points and $\tilde{\mathbf{N}}_{m 1}, \ldots, \tilde{\mathbf{N}}_{m N_{k}}$ represent the matrices correspond to the correlation between the common and the non-common points and $\mathbf{N}_{k}$ being the number of the non-common points. The right-hand term is defined as:

$\mathbf{u}=\left[\begin{array}{llll}\sum_{k=1}^{r} \mathbf{u}_{k}^{T} & \mathbf{u}_{1}^{T} & \cdots & \mathbf{u}_{r}^{T}\end{array}\right]^{T}$

The normal equation matrix has a rank deficiency equal to one. In fact any correct leveling network adjustment ends up with a singular covariance matrix due to the rank defect of the design matrix and consequently of the normal equations matrix. This is the algebraic consequence of the physical fact that observed 
height differences are invariant under changes of the height origin. The vertical datum remains undefined and height estimates are not unique. The final solution is derived through the inner constraints approach and it is expressed as follows:

$$
\delta \hat{\mathbf{h}}_{E}=\left(\mathbf{N}+\mathbf{1}_{N} \mathbf{1}_{N}^{T}\right)^{-1} \mathbf{u}
$$

where $N=m+N_{k}$ the total number of the points (common and non-common). We should notice here that the vector $\delta \hat{\mathbf{h}}_{E}=\left[\begin{array}{c}\delta \hat{\mathbf{h}}_{E}^{c} \\ \delta \hat{\mathbf{h}}_{E}^{k}\end{array}\right]$ refers to the estimated corrections which should be added to the approximate height values in order to derive the final estimated heights $\hat{\mathbf{h}}_{E}$. In addition, the vector $\delta \hat{\mathbf{h}}_{E}^{c}=\left[\begin{array}{lll}\delta \hat{h}_{E}^{c, 1} & \cdots & \delta \hat{h}_{E}^{c, m}\end{array}\right]^{T}$ corresponds to the $m$ common points of the networks, while the term $\delta \hat{\mathbf{h}}_{E}^{k}=\left[\begin{array}{lll}\delta \hat{h}_{E}^{1} & \cdots & \delta \hat{h}_{E}^{N_{k}}\end{array}\right]^{T}$ to the non-common points. The covariance matrix of the estimated points is:

$$
\mathbf{C}_{\mathbf{h}_{E}}=\left(\mathbf{N}+\mathbf{1}_{N} \mathbf{1}_{N}^{T}\right)^{-1}-\mathbf{1}_{N}\left(\mathbf{1}_{N}^{T} \mathbf{1}_{N}\right)^{-2} \mathbf{1}_{N}^{T}=\left(\mathbf{N}+\mathbf{1}_{N} \mathbf{1}_{N}^{T}\right)^{-1}-\frac{1}{N^{2}} \mathbf{1}_{N} \mathbf{1}_{N}^{T}
$$

An equivalent covariance matrix expression is:

$$
\mathbf{C}_{\mathbf{h}_{E}}=\left(\mathbf{N}+\mathbf{1}_{N} \mathbf{1}_{N}^{T}\right)^{-1} \mathbf{N}\left(\mathbf{N}+\mathbf{1}_{N} \mathbf{1}_{N}^{T}\right)^{-1}
$$

The rescaled covariance matrix will be:

$$
\boldsymbol{\Sigma}_{\mathbf{h}_{E}}=\hat{\sigma}^{2} \mathbf{C}_{\mathbf{h}_{E}}
$$

where the a posteriori variance factor is (e.g Angermann, 2004):

$$
\hat{\sigma}^{2}=\frac{\mathbf{b}^{T} \mathbf{P}_{E} \mathbf{b}-\mathbf{u}^{T} \delta \hat{\mathbf{h}}_{E}}{f}
$$

where $\mathbf{b}$ is the vector of the reduced observations (for each network $\mathbf{h}_{E}^{k}-\mathbf{h}^{o}$ where the superscript $o$ denotes the approximate values) and $f$ the number of redundant information (degree of freedom). An equivalent mathematical expression for the a posteriori variance factor can be written as:

$$
\hat{\sigma}^{2}=\frac{\mathbf{b}^{T} \mathbf{P}_{E} \mathbf{b}-\mathbf{u}^{T} \delta \hat{\mathbf{h}}_{E}}{d}
$$

where $d$ is the difference between the total number of observed heights at all networks and the number of the unknowns. The weight matrix $\mathbf{P}_{E}$ of the observations of all contributing networks is expressed as follows:

$$
\mathbf{P}_{E}=\left[\begin{array}{lll}
\mathbf{P}_{1} & & \\
& \ddots & \\
& & \mathbf{P}_{r}
\end{array}\right]=\left[\begin{array}{lll}
\mathbf{C}_{h_{1}^{E}}^{-1} & & \\
& \ddots & \\
& & \mathbf{C}_{h_{r}^{E}}^{-1}
\end{array}\right]
$$

where $\mathbf{C}_{h_{1}^{E}} \ldots \mathbf{C}_{h_{r}^{E}}$ is the error covariance matrix of the observed heights of each combined network.

However, the covariance matrix $\mathbf{C}_{h_{i}^{E}}$ is singular as already mentioned. In order to overcome the singularity, we add a relatively small positive quantity $\delta$ to the diagonal elements of the matrix (Bjerhammar, 1973; Sillard and Boucher, 2001). The new weight matrix will be constructed from the following alternative form:

$\tilde{\mathbf{C}}_{h_{i}^{E}}=\mathbf{C}_{h_{i}^{E}}+\delta \mathbf{I}$

$i=1 \ldots r$

$0<\delta \ll 1$

At this point we should summarize the aspects of our approach. It is optimal in the sense that the norm of the estimated heights becomes minimal. In other words the heights are estimated close to the a priori heights. At the same time the trace of the covariance matrix becomes minimal. In other words, we get in sum the best 
Table 1 The set of common stations, their heights and associated errors in the three examined vertical networks. Values are in meters.

\begin{tabular}{|c|c|c|c|}
\hline Common points & \#1 network & \#2 network & \#3 network \\
\hline $\mathrm{R} 1$ & $100.46 \pm 0.021$ & $100.550 \pm 0.003$ & $100.590 \pm 0.003$ \\
\hline $\mathrm{R} 2$ & $108.66 \pm 0.022$ & $108.75 \pm 0.003$ & $108.790 \pm 0.002$ \\
\hline R3 & $99.477 \pm 0.017$ & $99.578 \pm 0.004$ & $99.611 \pm 0.001$ \\
\hline $\mathrm{R} 4$ & $90.987 \pm 0.021$ & $91.061 \pm 0.003$ & $91.116 \pm 0.003$ \\
\hline R5 & $150.441 \pm 0.020$ & $150.530 \pm 0.005$ & $150.57 \pm 0.003$ \\
\hline R6 & $120.563 \pm 0.029$ & $120.640 \pm 0.006$ & $120.680 \pm 0.001$ \\
\hline R7 & $160.710 \pm 0.017$ & $160.83 \pm 0.004$ & $160.840 \pm 0.002$ \\
\hline $\mathrm{R} 8$ & $88.679 \pm 0.017$ & $88.776 \pm 0.005$ & $88.806 \pm 0.002$ \\
\hline R9 & $112.574 \pm 0.016$ & $112.671 \pm 0.003$ & $112.700 \pm 0.001$ \\
\hline $\mathrm{R} 10$ & $97.665 \pm 0.011$ & $97.747 \pm 0.004$ & $97.797 \pm 0.003$ \\
\hline
\end{tabular}

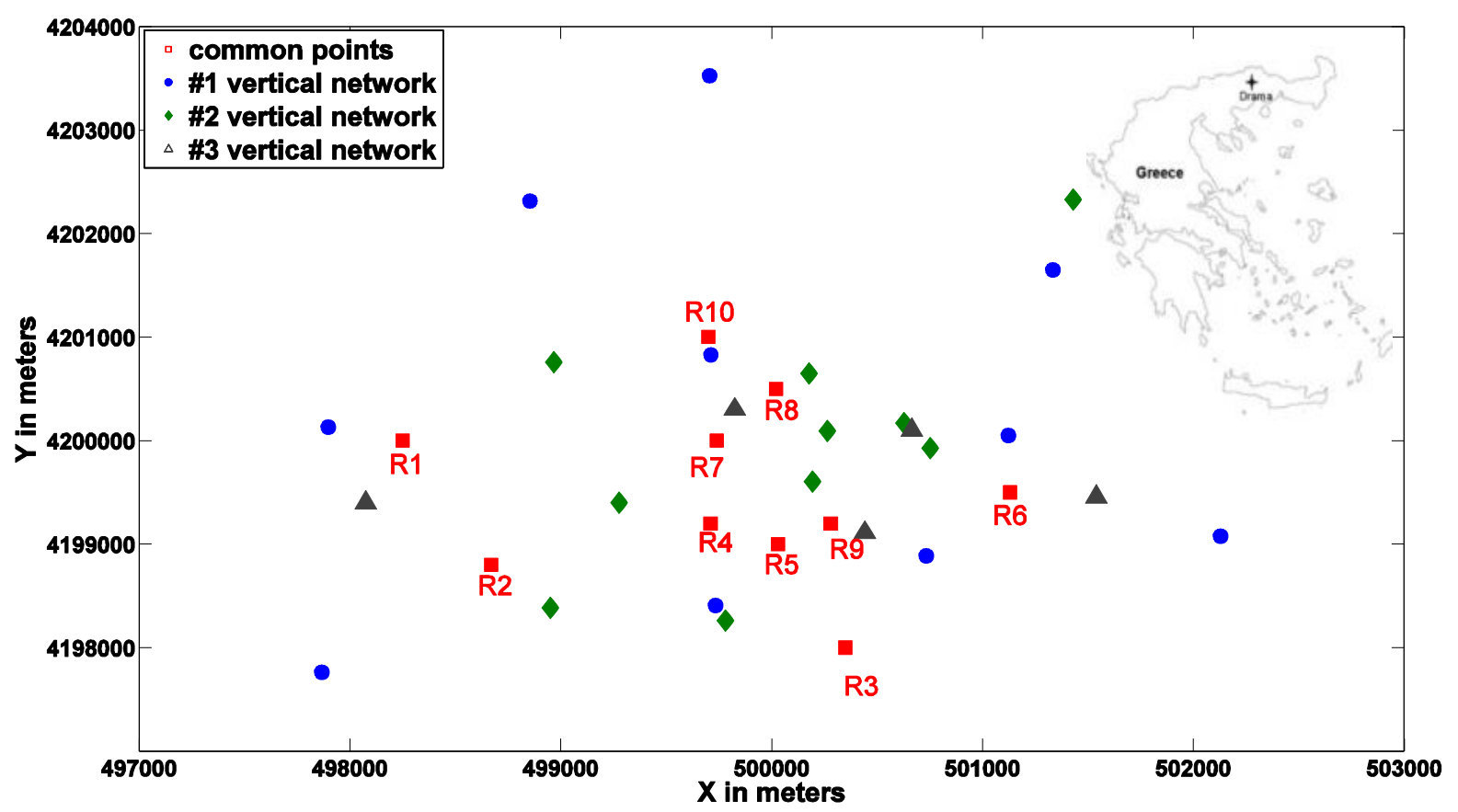

Fig. 1 The examined vertical networks and their location in Greece.

accuracies of all height estimates. This could be very useful when combining old height determinations (which in many cases were measured with low accuracy methods, e.g, trigonometric leveling) or/and in cases of inconsistencies due to different datum definitions show up. We should here emphasize that the proposed approach does not address the absolute level of the adjusted network. It handles solely the estimation of relative datum offsets between the participating networks, based entirely on the available accuracy estimates of the existing height values.

When we are dealing with old leveling studies it is true that only variances are given and not the covariances (diagonalized covariance matrix). However, in such studies, there is a drawing of the levelling network indicating the observed network loops. Thus, it is trivial to reconstruct the design matrix of the network, If the loop lengths are known, even approximately, the weight matrrix $\mathbf{P}$ can be constructed using one of the standard weighting schemes for leveling. Then the Normal Equation matrix is directly computed, and we proceed with the computation of the right hand term $\mathbf{u}$ and the combination of the Normal Equations. Nevertheless, it is not guaranteed that the assumed loops coincide with the consisting networks geometry.

\section{NUMERICAL IMPLEMENTATION}

The proposed approach was implemented and tested with real world data, using three different leveling networks located in the Northern part of Greece close to the city of Drama (Fig. 1). The first and the second network consist of 20 stations each, the third of 15 respectively. These three leveling networks share 10 common stations. Solely standard deviations are available to denote the accuracies of the points. Furthermore the datum definitions are unknown. Table 1 presents the height estimates and 
Table 2 The approximated and adjusted common stations heights along with their associated standard errors. Values are in meters.

\begin{tabular}{ccc}
\hline station & app. value & est. value and error \\
\hline R1 & 100.569 & $100.528 \pm 0.003$ \\
R2 & 108.777 & $108.730 \pm 0.002$ \\
R3 & 99.609 & $99.553 \pm 0.003$ \\
R4 & 91.087 & $91.052 \pm 0.002$ \\
R5 & 150.557 & $150.512 \pm 0.003$ \\
R6 & 120.679 & $120.635 \pm 0.003$ \\
R7 & 160.837 & $160.789 \pm 0.002$ \\
R8 & 88.800 & $88.752 \pm 0.003$ \\
R9 & 112.697 & $112.652 \pm 0.003$ \\
R10 & 97.774 & $97.738 \pm 0.002$ \\
\hline
\end{tabular}

Table 3 The residuals statistics.

\begin{tabular}{lcc}
\hline & statistical quantity & value in $\mathrm{mm}$ \\
\hline $\min$ & -28.3 \\
$\max$ & 28.1 \\
average & 0.0 \\
standard deviation & 9.3 \\
\hline
\end{tabular}

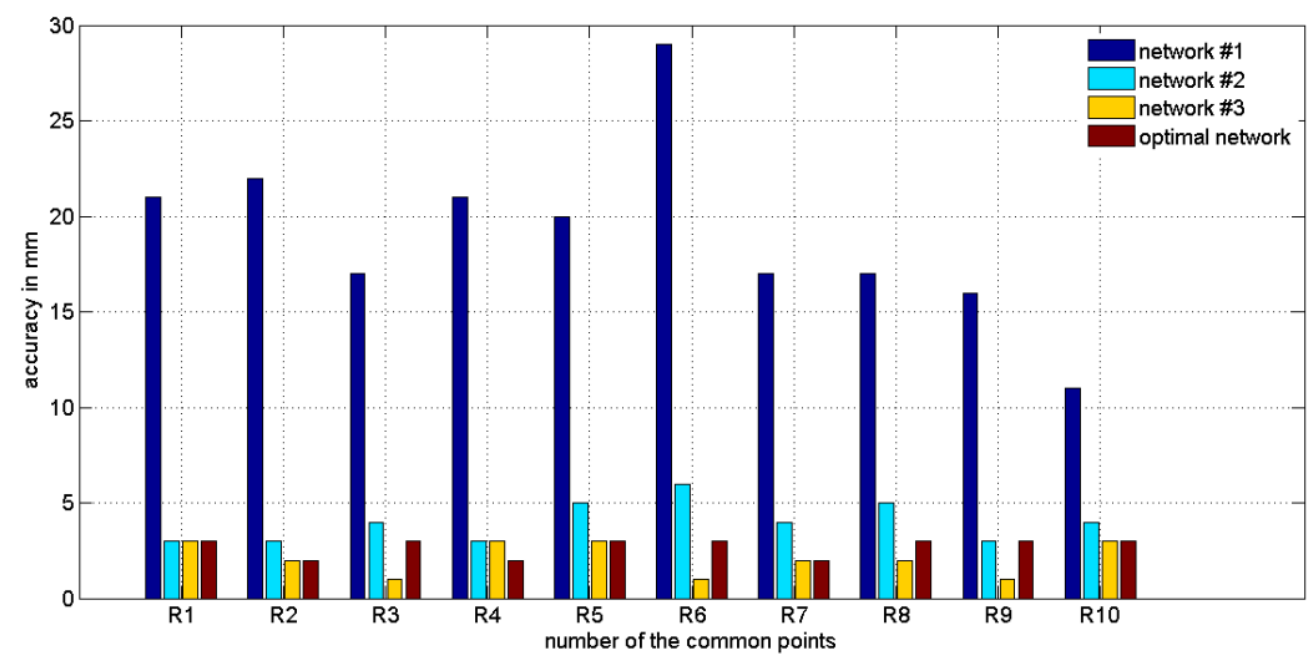

Fig. 2 The standard deviations of the heights of the common stations with respect to each network.

the associated accuracies for each one of the three networks. Figure 1 depicts the network of common points.

The first network was established in the early 1980s using trigonometric leveling (blue circles). The second was realized in the late 1990s (green diamonds) and the third one (grey triangles) in 2005 respectively (both with spirit leveling). At first glance, there are obvious offsets between the networks (e.g the first and the third networks have a discrepancy of $\sim 13 \mathrm{~cm}$ ). This offset could be a consequence of the different datum definitions among the networks (official vertical datum versus the height datum of the triangulation benchmarks). In addition, the estimated heights from trigonometric leveling could possibly be affected by some systematic errors. The standard errors of the non-common points vary from 11 to $35 \mathrm{~mm}$ for the first network, 1 to $6 \mathrm{~mm}$ for the second network and 1 to $4 \mathrm{~mm}$ for the third one, respectively. The degree of freedom is 20 (55 observed minus 35 unknown heights).

The least squares adjustment for the optimal reference network realization is laid out in Table 2 . The approximate heights are estimated for each station from the observed heights weighted mean average (weights equal to the reciprocal error variance of the height with respect to each network). The associated standard errors were estimated from the re-scaled covariance matrix of the unknown parameters (Eq. 16). Figure 3 visualizes the standard errors of the 


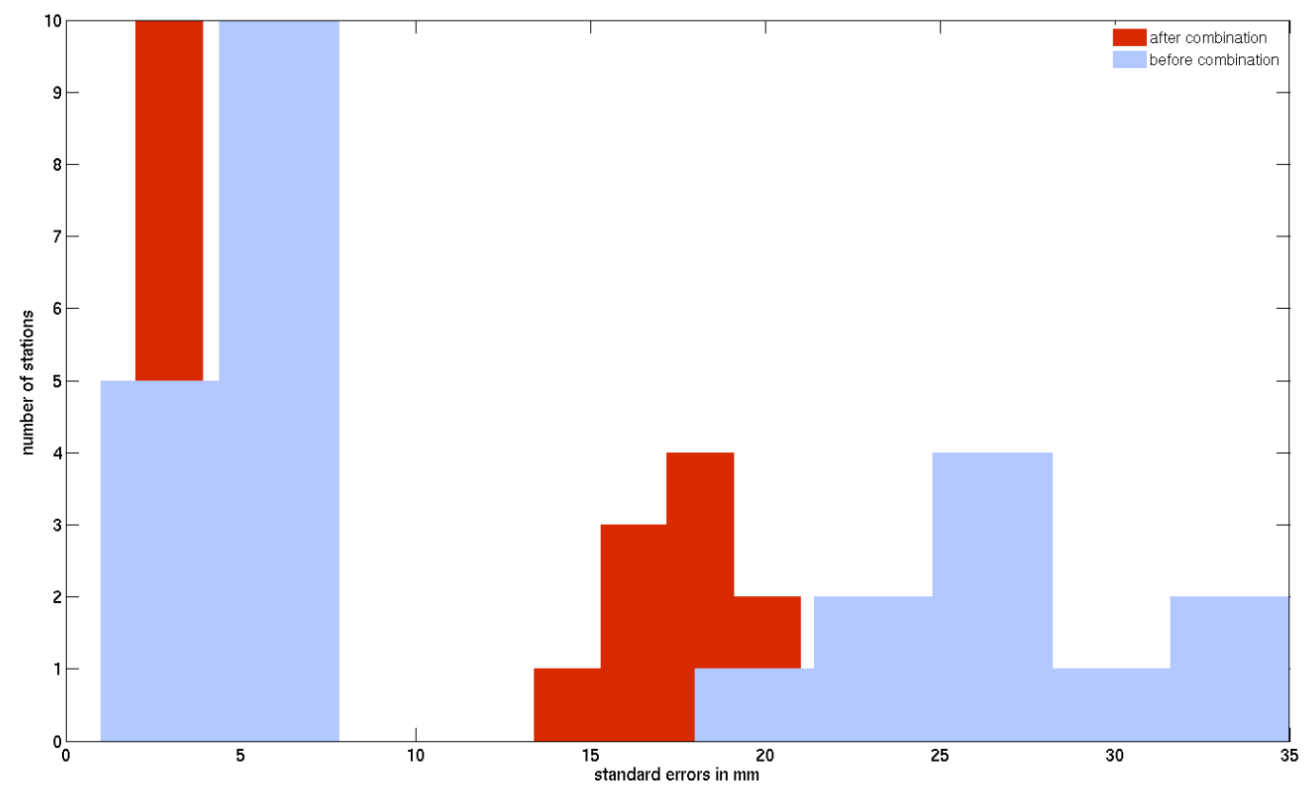

Fig. 3 The histograms of the standard errors before and after the combination.

Table 4 The external validation results. Values are in meters for the differences and in $\mathrm{km}$ for the leveling length.

\begin{tabular}{ccccc}
\hline from $\square$ to & measured difference & estimated difference & leveling length & discrepancy \\
\hline $\mathrm{R} 1-->\mathrm{R} 2$ & $8.202 \pm 0.003$ & $8.201 \pm 0.002$ & 0.85 & $0.001 \pm 0.003$ \\
$\mathrm{R} 3-->\mathrm{R} 4$ & $-8.501 \pm 0.003$ & $-8.501 \pm 0.001$ & 2.42 & $0.000 \pm 0.003$ \\
$\mathrm{R} 9-->\mathrm{R} 10$ & $-14.914 \pm 0.003$ & $-14.912 \pm 0.002$ & 1.15 & $-0.002 \pm 0.003$ \\
\hline
\end{tabular}

unknown parameters. The a posteriori variance factor is estimated $1.2845 \mathrm{~mm}^{2}$. We also apply a $3-\sigma$ criterion for outlier rejection $(\sigma=9.3 \mathrm{~mm})$ and found that all the observations pass it. Table 3 presents the residuals statistics.

Firstly we can observe that for the common points we obtain standard errors up to $3 \mathrm{~mm}$. The accuracies differ 1-2 $\mathrm{mm}$ from the most accurate solution (third network). At the same time, we have a clear improvement of the accuracies of the first network. Another interesting fact is the improvement of the standard deviations for the non-common stations. For the first network the standard deviations are improved from 7 to $21 \mathrm{~mm}$, for the second by 1 to $5 \mathrm{~mm}$ and for the third by 2 to $4 \mathrm{~mm}$, respectively.

The combination of the different height networks decreases the quality of some of the most accurate heights to be found in the third network at the level of $1-2 \mathrm{~mm}$. One should note that our aim is to handle all the existing information based on all 35 benchmarks. This alternative computational scheme improves the standard deviations of the common points of the first network by $10 \mathrm{~mm}$. For the second network, the height standard deviations of the heights remain practically at the same level, while for the third one, we obtain slightly worse standard errors. Before the combination, the mean height standard error was $13.5 \mathrm{~mm}$, while after the combination becomes $8.6 \mathrm{~mm}$. Figure 3 visualizes the histograms of the distribution of the standard deviations before and after the combination, respectively. This practically demonstrates that the implementation of the alternative strategy's leads to an improvement at the level of $5 \mathrm{~mm}$ for the standard deviations of the more accurate points. The less accurate points see an improvement of $10 \mathrm{~mm}$ and more.

For the completeness of our analysis we measured on hindsight three randomly selected height differences out of the common points using spirit leveling measurements. We examined the quality of the measured height differences against the estimated height differences from the optimal vertical network. For the leveling observations we used the Leica DNA03 instrumentation (accuracy: $1 \mathrm{~mm} / \mathrm{km}$ ). We measured the height differences using double leveling. The standard errors of the observations were $3 \mathrm{~mm}$. Table 4 presents the validation results and the associated standard deviations. 
The results show that the estimated height differences of the optimal network agree within $3 \mathrm{~mm}$ with the observed height differences.

\section{CONCLUSIONS AND FURTHER INVESTIGATIONS}

The new approach is designed for the optimal combination of different height information from different vertical network realizations in a specified area. Optimal means that the adjusted heights will have minimum norm with respect to the a priori heights and minimum trace of their covariance matrix. The mathematical tool of this methodology is based on the reconstruction of the normal equations and implementing the inner constraints approach. The new methodology was tested in a local leveling network with three independent realizations by different techniques, having 10 common points. The new standard errors for the common points are found at the level of 2-3 $\mathrm{mm}$ which is close to the accuracy of the best contributed network (in terms of its initial accuracy). Meanwhile, the non common points standard errors of the first network is improved, while on the second one the standard deviations remain at the same level. The improvement of the mean standard error of the non-common points is found at the level of $5 \mathrm{~mm}$, as it is shown in Figure 3. The third network's accuracy does not seem to be improved. However, we shall again address that our main concern is to exploit all the existing height information, not only the common points among the networks. Finally, three exemplary height differences have been re-measured by spirit leveling with millimeter accuracy which showed no significant differences to the estimates.

The new approach can be beneficially adopted for the densification of existing leveling networks. Thus, time and cost consuming re-measurements can be avoided. The proposed methodology can easily be expanded to include GNSS-leveling networks. Also the approach can be migrated by temporal evolution terms to account for time-dependent systematics in vertical networks.

\section{ACKNOWLEDGMENTS}

Professor Athanasios Dermanis and the two anonymous reviewers are kindly acknowledged for their contributions which led to a significant improvement of the initial manuscript.

\section{REFERENCES}

Angermann, D., Drewes, H., Krügel, M., Meisel, B., Gerstl, M., Kelm, R., Müller, H., Seemüller, W. and Tesmer, V.: 2004, ITRS Combination Centre at DGFI - A terrestrial reference frame realization 2003. Deutsche Geodätische Kommission, Reihe B, Nr. 313, München.
Bjerhammar, A.: 1973, Theory of errors and generalized matrix inverses. Amsterdam, Elsevier.

Blaha, G.: 1971, Inner adjustment constraints with emphasis on range observations. Department of Geodetic Science. The Ohio State University, Report No. 148. Columbus, Ohio.

Dermanis, A.: 1987, Observations adjustment and estimation theory. Thessaloniki, Zete Publications, (in Greek).

Fotopoulos, G.:2003, An analysis on the optimal combination of geoid, orthometric and ellipsoidal height data. PhD Dissertation, Department of Geomatics, University of Calgary, Canada.

Hirt, C., Schmitz, M., Feldmann-Westendorff, U., Wübbena, G., Jahn, C.-H. and Seeber, G.: 2011, Mutual validation of GNSS height measurements and highprecision geometric-astronomical leveling. GPS Solutions, 15 , No. 2, 149-159. DOI: $10.1007 / \mathrm{s} 10291-010-0179-3$

Hofmann-Wellenhof, B. and Moritz, H.: 2005, Physical Geodesy. Berlin Heildeberg, Springer.

Koch, K.-R.: 1999, Parameter Estimation and Hypothesis Testing in Linear Models, second edition. Berlin Heidelberg, Springer.

Kotsakis, C. and Sideris, M.G.: 1999, On the adjustment of combined GPS/leveling/geoid networks. J. Geod., 73, No. 8, 412-421. DOI: 10.1007/s001900050261

Kotsakis, C., Katsambalos, K. and Ampatzidis, D.: 2012, Estimation of the zero-height geopotential level in a local vertical datum from inversion of co-located GPS, leveling and geoid heights: a case study in the Hellenic islands. J. Geod., 86, No. 6, 423-439. DOI: $10.1007 / \mathrm{s} 00190-011-0530-7$

Sillard, P. and Boucher, C.: 2001, A review of algebraic constraints in terrestrial reference frame datum definition. J. Geod., 75, 63-71. DOI: $10.1007 / \mathrm{s} 001900100166$

Torge, W: 2001, Geodesy, third edition. Berlin, de Gruyter.

Vanicek, P. and Krakiwsky, E.: 1982, Geodesy: The Concepts. New York: North-Holland Publishing Company.

Wang, Y.M., Saleh, J., Li, X. and Roman, D.R.: 2012, The US gravimetric geoid of 2009 (USGG2009): Model development and evaluation. J. Geod., 86, No. 3, 165-180. DOI: 10.1007/s00190-011-0506-7 